\title{
AIN and Al oxy-nitride Gate Dielectrics for Reliable Gate Stacks on Ge and InGaAs channels
}

Y Guo, H Li, J Robertson

Engineering Dept, Cambridge University, Cambridge CB2 1PZ, UK

\begin{abstract}
AlN and $\mathrm{Al}$ oxy-nitride dielectric layers are proposed instead of $\mathrm{Al}_{2} \mathrm{O}_{3}$ as a component of the gate dielectric stacks on higher mobility channels in metal oxide field effect transistors to improve their positive bias stress instability (PBTI) reliability. It is calculated that the gap states of nitrogen vacancies in AlN lie further away in energy from the semiconductor band gap than those of oxygen vacancies in $\mathrm{Al}_{2} \mathrm{O}_{3}$, and thus $\mathrm{AlN}$ might be less susceptible to charge trapping and have a better reliability performance. The unfavourable defect energy level distribution in amorphous $\mathrm{Al}_{2} \mathrm{O}_{3}$ is attributed to its larger coordination disorder compared to the more symmetrically bonded AlN. Al oxy-nitride is also predicted to have less tendency for charge trapping.
\end{abstract}

\section{Introduction}

A considerable fraction of the performance increase of complementary metal oxide semiconductor (CMOS) devices in the last 10 years has come from introducing new materials, rather than from dimensional scaling itself [1]. One of the major changes was the replacement of the $\mathrm{SiO}_{2}$ gate dielectric by the high dielectric constant (high $\mathrm{K}$ ) oxide $\mathrm{HfO}_{2} . \mathrm{HfO}_{2}$ was chosen primarily due to its thermal stability and band offset [2]. A further advantage was that the presence of a thin $\mathrm{SiO}_{2}$ interfacial layer between the $\mathrm{Si}$ channel and the $\mathrm{HfO}_{2}$ helped to give a good interface with a low density of gap states, $D_{\text {it }}$ [3-5]. Finally, the energy levels of the $\mathrm{O}$ vacancy in $\mathrm{HfO}_{2}$, its main defect, were sufficiently far away in energy from the Si band gap, that their impact on device reliability was not too large [4-6]. In contrast, another contender high $\mathrm{K}$ oxide, $\mathrm{Al}_{2} \mathrm{O}_{3}$, was not chosen because of its lower $\mathrm{K}$ value and higher density of traps [7-10].

To continue scaling of CMOS devices, it is now desired to replace Si channels with a higher mobility semiconductors like Ge or a III-V semiconductor [11,12]. Generally, the existing gate stack designs for Ge or III-Vs have been optimised to minimise the defect density on the semiconductor side of the interface. Ge has a much higher electron and hole mobility than Si, and it would be the simplest solution. However, the $\mathrm{Ge} / \mathrm{GeO}_{2}$ interface has a much higher density of interface states, which appear to be linked to O deficiency [11-15]. These defects are believed to arise from the diffusion of $\mathrm{O}$ vacancies through the $\mathrm{GeO}_{2}$ layer to the interface [15-16]. In addition, $\mathrm{HfO}_{2}$ was often avoided as a component of gate stacks on Ge because of reaction between $\mathrm{HfO}_{2}$ and the $\mathrm{Ge}$ or $\mathrm{GeO}_{2}$ [13]. Thus, a $\mathrm{Al}_{2} \mathrm{O}_{3}$ layer is often included in gate stacks on Ge to act as an oxygen diffusion barrier to prevent degradation of the Ge/oxide interface [17-20]. Similarly, $\mathrm{Al}_{2} \mathrm{O}_{3}$ is usually included in the gate stack design on GaAs or InGaAs [21-26, to act as an oxygen diffusion barrier, inhibit the oxidation of the underlying III-V, inhibit the preferential oxidation of the Ga sites [27] and thus the injection of excess As into the semiconductor as $\mathrm{As}_{\mathrm{Ga}}$ antisites, whose associated deep levels are seen experimentally [28].

However, it is now known that these gate stacks on Ge or III-Vs have worse reliability problems that those on Si. The gate stacks suffer from a charge trapping at existing defects in their gate dielectrics, which shifts the gate threshold voltage $V_{t}$ [29-34]. This degradation is 
known as positive or negative bias instability (PBTI, NBTI). Recent extensive measurements of Franco et al [30,31] and Groesenken et al [32] have shown how the PBTI and NBTI behaviour of $\mathrm{Ge}$ and III-V based FETs is worse than for the equivalent Si devices, Fig 1(a). The charge trapped in defects in the gate dielectric in PBTI or NBTI, $\Delta \mathrm{N}$, can be measured as a function of the gate over-drive voltage, $\Delta \mathrm{V}_{\mathrm{g}}=\mathrm{V}_{\mathrm{g}}-\mathrm{V}_{\mathrm{t}}$ [4,5], where $\mathrm{V}_{\mathrm{t}}$ is the gate threshold voltage. $\Delta \mathrm{N}$ is found to vary with $\Delta \mathrm{V}_{\mathrm{g}}$ as a power law, $\Delta \mathrm{N}=\mathrm{a} . \Delta \mathrm{V}^{\gamma}$, where the exponent $\gamma$ is known as the voltage acceleration factor [4]. The size of $\gamma$ can be used to identify the BTI mechanism and the nature of the defect states which trap the charge. The PBTI process in Ge and III-V nFETs was found to have the same $\gamma$ value $[31,32]$, which points to a common mechanism. This mechanism was identified as a charge trapping in existing border traps in the $\mathrm{Al}_{2} \mathrm{O}_{3}$ layer [30-34], due to the presence of $\mathrm{Al}_{2} \mathrm{O}_{3}$ in both cases. It was thus concluded that gate stacks on Ge and III-Vs must be improved to achieve the necessary 10 year reliability lifetime, requiring a modification or removal of the $\mathrm{Al}_{2} \mathrm{O}_{3}$. In retrospect, it has become clear that Ge or III-V devices should be optimised on the basis of good reliability, not just a low $\mathrm{D}_{\mathrm{it}}$ of the initial devices.

The low value of $\gamma$ for Ge and III-V devices indicates that the defect band causing the PBTI problem lies above the conduction band edge of the semiconductor, but that its states have a broad energy distribution that tails down into the semiconductor's band gap [31,32], Fig 1(b). Thus, the gate stack must be modified to use a dielectric whose defect levels lie further away energetically from the semiconductor band gap, and with a narrower energy distribution. Thus, we calculate the defect energy states of $\mathrm{Al}_{2} \mathrm{O}_{3}$ and related dielectrics to see if improvements are possible.

\section{Methods}

The calculations are carried out using the plane wave density functional code CASTEP. The structural calculations on large cells use the generalised gradient approximation (GGA) of the exchange-correlation functional. In order to correct the GGA band gap error, the electronic structures are calculated within the screened exchange (sX) functional [35], with norm conserving pseudopotentials and a plane wave cutoff energy of $750 \mathrm{eV}$. The defect formation energies are calculated using the method of Lany and Zunger [36] using their corrections.

The electronic structure of an amorphous random network depends primarily on the local order, of which the coordination number is the most important parameter [37]. Thus, one can calculate the local electronic structure of bulk sites using a random network model, or by using a suitable crystalline polymorph that has similar local order, such as the ST12 Si phase for a-Si, to lower computational costs.

The calculation of defects in these networks depends on the valence. Oxygen has an even number of electrons so it turns out that one can consider the oxygen excess or deficiency in say $\mathrm{SiO}_{2}$ in terms of $\mathrm{O}$ interstitials or vacancies or in terms of $\mathrm{Si}-\mathrm{Si}$ bonds or O-O bonds. This also applies to $\mathrm{Al}_{2} \mathrm{O}_{3}$. $\mathrm{O}$ excess or deficiency can be expressed in terms of $\mathrm{O}$ interstitials or vacancies, and the most stable charge states are spin-paired. Thus it is safe to use polymorphs as models of the local $\mathrm{Al}_{2} \mathrm{O}_{3}$ structure even for its defects.

Specifically, for a- $\mathrm{Al}_{2} \mathrm{O}_{3}$ we use a supercell based on a suitable crystalline polymorph of $\mathrm{Al}_{2} \mathrm{O}_{3}$. Amorphous $\mathrm{Al}_{2} \mathrm{O}_{3}$ prepared by atomic layer deposition (ALD) is characterised by a network of different coordinations, with $67 \%$ of the Al sites being 4-fold [38,39], a mass density somewhat less than sapphire's, and an optical band gap of $\sim 6.5 \mathrm{eV}$ [40]. Molecular dynamics simulations of $\mathrm{a}-\mathrm{Al}_{2} \mathrm{O}_{3}$ find $\mathrm{Al}$ and $\mathrm{O}$ coordinations similar to those measured in experiment 
[41]. Sapphire $\left(\alpha-\mathrm{Al}_{2} \mathrm{O}_{3}\right)$ is a poor model of a- $\mathrm{Al}_{2} \mathrm{O}_{3}$ because it has too high an atomic density, $100 \%$ of its $\mathrm{Al}$ sites are octahedral (6-fold), and its band gap of $8.8 \mathrm{eV}$ is too large. $\kappa-\mathrm{Al}_{2} \mathrm{O}_{3}$ has been used as a possible model for $\mathrm{a}-\mathrm{Al}_{2} \mathrm{O}_{3}[42,43]$. However, this polymorph still contains $75 \%$ of 6-fold $\mathrm{Al}$ sites and $25 \%$ of 4-fold $\mathrm{Al}$ sites, and it has a $~ 8.0 \mathrm{eV}$ band gap [44,45], which is 1.5 $\mathrm{eV}$ too large. Hence here, as previously [46], we use the $\theta-\mathrm{Al}_{2} \mathrm{O}_{3}$ polymorph with the $\beta-\mathrm{Ga}_{2} \mathrm{O}_{3}$ structure. It has $50 \%$ of 4 -fold $\mathrm{Al}$ sites, $50 \%$ of 6 -fold sites, and a similar density to a- $\mathrm{Al}_{2} \mathrm{O}_{3}$ $[47,48]$. Its calculated band gap is $6.5 \mathrm{eV}$ [46], close to the experimental value for a- $\mathrm{Al}_{2} \mathrm{O}_{3}[40]$. Thus our calculations on oxygen vacancies are made on periodic supercells of 120 atoms of $\theta$ $\mathrm{Al}_{2} \mathrm{O}_{3}$.

For a-AlN, we use two methods to study the defects. First we consider N vacancies in periodic wurzite supercells of 96 atoms. We note that because of valence, the neutral $\mathrm{N}$ vacancy has a unpaired spin and so is paramagnetic, whereas a-AlN is generally diamagnetic. We therefore also consider random network models of a-AlN. We constructed stoichiometric 96 atom random network models of a-AlN by molecular dynamics using CASTEP. The system is annealed at $3000 \mathrm{~K}$ for $10 \mathrm{ps}$, then quenched at $10 \mathrm{~K} / \mathrm{ps}$ to $300 \mathrm{~K}$, and then the geometry is relaxed to an energy minimum. As in Chen and Drabold [49], we find the networks to contain a considerable fraction of 3-fold bonded $\mathrm{Al}$ and $\mathrm{N}$ sites. These models were then used as the basis in which to form defects.

Finally, ten different defect random network models of a-AlON $\mathrm{x}_{\mathrm{x}}$ were generated, with 100-200 atoms, to model different compositions and positions of the vacancy.

\section{Results and Discussion}

The calculated formation energies of the $\mathrm{O}$ vacancies in $\theta-\mathrm{Al}_{2} \mathrm{O}_{3}$ are shown in Fig. 2(a) as a function of Fermi energy. The charge states of each line are labelled. The most stable charge state has the lowest formation energy at that value of Fermi energy. The transition energies, where the stable charge state changes, are summarised in Fig. 3. The transition energies are aligned to the band edge energies of $\mathrm{Ge}$ and $\mathrm{In}_{0.53} \mathrm{Ga}_{0.47} \mathrm{As}$ using band alignments calculated previously [50]. We see that there are two empty defect levels above midgap and two filled levels below midgap. Both of the energy levels lie adjacent to the conduction bands (CB) and valence bands (VB) of the Ge or III-V channel.

In $\theta-\mathrm{Al}_{2} \mathrm{O}_{3}$, there are two different oxygen sites, with different arrangements of 4-fold or 6-fold $\mathrm{Al}$ neighbours around them. This leads to Pauling bond orders at the $\mathrm{O}$ sites which differ from the expected value of two. This causes a strong disorder at the $\mathrm{O}$ site in $\theta-\mathrm{Al}_{2} \mathrm{O}_{3}$. The low symmetry of the $\mathrm{O}$ vacancies splits its transition states into 2 states. This high degree of disorder at the $\mathrm{O}$ sites substantially broadens the vacancy energy levels in $\theta-\mathrm{Al}_{2} \mathrm{O}_{3}$.

Fig 3 also includes the transition levels of $\kappa-\mathrm{Al}_{2} \mathrm{O}_{3}$ as calculated by Choi [43]. Here we see that there is one defect level that lies around the band gap energy of Ge. This indicates that $\mathrm{O}$ vacancies in a- $\mathrm{Al}_{2} \mathrm{O}_{3}$ at sites surrounded mainly by 6-fold $\mathrm{Al}$ sites might give states within the $\mathrm{Ge}$ gap energy range.

In view of the defect state energies of $\mathrm{a}-\mathrm{Al}_{2} \mathrm{O}_{3}$, we consider other dielectrics such as a-AlN. The a-AlN would be made by ALD or by post plasma nitridation. Its preparation would still use tetra-methyl aluminium (TMA) which plays a critical role during deposition of removing surface As-As dimers which are a main source of interface states on III-V's [51-54]. Nitridation of the III-V interface is already known to reduce $\mathrm{D}_{\text {it }}$ on the semiconductor side, both theoretically [55] and experimentally [56,24], by moving the states out of the semiconductor's band gap. Here, we 
are proposing to use amorphous AlN as part of the dielectric, to move its defect states further away from the semiconductor's band gap range. This has already been shown experimentally by Luc et al [57].

The principle of using AlN is to have a wide band gap amorphous capping layer, but whose defect states might lie outside the critical energy range. We first considered the crystalline supercell model. The transition states for the $\mathrm{N}$ vacancy in c-AlN are shown in Fig 2(b). These states are related to those of $\mathrm{GaN}$ [58] and similar to those recently calculated by Varley et al [59]. We see that its transition levels lie further away from the Ge or InGaAs band gap energy range that those of the $\mathrm{O}$ vacancy in $\mathrm{Al}_{2} \mathrm{O}_{3}$. The energy levels have a narrower energy distribution than those of $\mathrm{Al}_{2} \mathrm{O}_{3}$. This is because the tetrahedral sites are more symmetric.

However, the structure of a-AlN is more complicated than the crystalline model. Fig 4(a) shows a 96 atom stoichiometric random network model generated by molecular dynamics. It contains only Al-N bonds and no like-atom bonds. Of the 96 atoms, in this case 10 of the atoms are 3 -fold coordinated and the rest are 4-fold coordinated (with equal numbers of Al and N's of each type). This is a sizable fraction of 3 -fold sites, and is consistent with the view that 3 -fold sites are common in amorphous III-V networks, because they can be formed as III and V atom pairs $[49,53,60]$.

Fig. 4(b) shows the partial density of states (PDOS) on the 4-fold and 3-fold sites for the bulk network, as calculated using the sX functional. It has a clean $5.4 \mathrm{eV}$ band gap on both types of sites, less than the $6.2 \mathrm{eV}$ of the crystal, but nonetheless a wide gap material. The 3-fold sites in a-AlN are similar to those on the (110)GaAs surfaces. The Al sites relax towards a planar geometry so their dangling bond state becomes empty and rises in energy to be in the conduction band. The $\mathrm{N}$ dangling bond states lie at the top of the valence band, and are filled. Thus both sets of dangling bond states lie outside the band gap. There is a charge transfer from 3-fold Al to 3fold $\mathrm{N}$ to ensure this occupancy. Because both the trivalent $\mathrm{Al}$ and trivalent $\mathrm{N}$ sites are satisfying their normal valence requirements, this is a low energy 'defect pair' of the tetrahedral network $[53,60]$.

We now create an $\mathrm{N}$ vacancy in this 96 atom network. Because $\mathrm{N}$ has 5 valence electrons, a single $\mathrm{N}$ vacancy would be spin unpaired. A realistic diamagnetic model would require two vacancies, which increases the network size and calculation cost. Instead, we form one vacancy and add one hydrogen atom for electron counting purposes, to keep a spin-paired network. The electronic structure is calculated within sX. The vacancy reconstructs to form a defect complex containing more 3-fold Al sites. The resulting network shows states within the gap. The local PDOS on the defect sites compared to bulk sites as reference is shown in Fig 5(a). We use these localised states to identify the position and nature of the defect. We find a filled defect state in the lower gap at $+1.3 \mathrm{eV}$ and an empty defect state in the upper gap at $+4.1 \mathrm{eV}$. The filled defect state for the neutral configuration is singly degenerate with a p-like symmetry and localised on one $\mathrm{N}$ atom, Fig 5(b). The empty state is localised on Al sites and is more delocalised. Overall there is a state-free gap of $2.8 \mathrm{eV}$, around the band gap energy range of Ge or InGaAs. Further discussion of the defect states of amorphous III-V solids will be given elsewhere.

These results suggest that a-AlN would form a suitable component of an improved gate stack. The lowest empty defect state lies higher against the CB than in the oxide, and the highest filled defect state lies lower against the VB in Figs 3,4, suggesting that AlN should improve both PBTI and NBTI in both PMOS and NMOS respectively.

In practice, pure a-AlN pure may not be formed; $\mathrm{Al}$ oxy-nitride might be formed, because of the susceptibility of AlN to oxidation. We have therefore also studied the case of anion defects in 
$\mathrm{AlON}_{\mathrm{x}}$ of various compositions. We have constructed random networks of $\left(\mathrm{Al}_{2} \mathrm{O}_{3}\right)_{\mathrm{x}}(\mathrm{AlN})_{1-\mathrm{x}}$ of 100-200 atoms, such as Fig 5(a). The compositions of the models are constructed to contain only Al-O and Al-N bonds, with their chemical preferred valence. There are no N-O bonds or N-N bonds. This differs from experiment in that $\mathrm{N}-\mathrm{O}$ bonds are sometimes found [61]. We then created $\mathrm{O}$ vacancies at 10 different sites, in order to check the repeatability. The vacancies can behave differently because of the different local compositions, but overall the defect states are still well away from the Ge band gap. Fig. 5(b) shows the local density of states on Al sites adjacent to one of these vacancies. Figs. 5(c,d) show the wavefunctions of the filled state and the empty state of the defect. We see that, as in the case of AlN itself, the vacancy states are away from the Ge or InGaAs band gap. We note also that they are sinlgy degenerate.

A range of other cases are found. They generally have the same energy spectrum of defect states, with states avoiding the Ge or InGaAs band gap region. This suggests that the anion site disorder in $\mathrm{AlON}_{\mathrm{x}}$ is also lower than in $\mathrm{Al}_{2} \mathrm{O}_{3}$. Interestingly in one case, it is possible to create an $\mathrm{O}$ vacancy in one part of the network, but after relaxation of the random network, the actual defect wavefunction localized itself away from where the vacancy was initially formed, but its defect orbital is still localised mainly on close Al sites.

\section{Conclusions}

In summary, the traps in $\mathrm{Al}_{2} \mathrm{O}_{3}$ based gate stacks were previously known to cause the positive bias instability in Ge and III-V MOSFETs, due to the tailing of their defect energy distribution towards the semiconductor band gap. We show that the defect causing the problem is the $\mathrm{O}$ vacancy. It is then shown that $\mathrm{AlN}$ or $\mathrm{AlON}_{\mathrm{x}}$ based gate stacks should have a better reliability because they have fewer vacancy states in the energy range of the critical semiconductor band gap region. This arises because AlN and AlOx have less disorder to broaden the vacancy levels. Creation of a $\mathrm{N}$ vacancy in a-AlN leads to a defect complex.

\section{Acknowledgements}

The authors acknowledge funding from EPSRC. 


\section{References}

1. K J Kuhn, Tech Digest IEDM (IEEE, 2012) p 8.01

2. J Robertson, Rep Prog Phys (2006); J Robertson, R M Wallace, Mat Sci Eng R 881 (2015)

3. T Ando, Materials, 5478 (2012)

4. A Kerber, E A Cartier, IEEE Trans Device Mater Reliability 9149 (2009)

5. $\quad$ E Cartier et al, Tech Digest IEDM (2013) p18.4

6. L A Ragnarsson, T Chiarella, M Togo, T Schram, P Absil, T Hoffman, Microelec Eng 88 1317 (2011)

7. $\quad$ S Zafar, A Callegari, J Appl Phys 939298 (2003)

8. $\quad$ M Specht, M Stadele, S Jaschik, U Schroder, App Phys Lell 843076 (2004)

9. C C Yeh, T P Ma, N Ramaswamy, N Rocklein, D Gealy, T Graettinger, K Min, App Phys Lett, 91113521 (2007)

10. L S Salomone, J Lipovetzky, S H Carbonetto, M A G Inza, E G Redin, F Campabadal, A Faigon, J App Phys Lett 113074501 (2013)

11. S Takagi, R Zhang, J Suh, S H Kim, M Yokoyama, K Nishi, M Takenaka, Jpn J App Phys 54 06FA01 (2015)

12. A Toriumi, C H Lee, S K Wang, T Tabata, M Yoshida, D D Zhao, T Nishimura, K Kita, K Nagashio, Tech Digest IEDM (IEEE, 2011)

13. Y Kamata, Materials Today 1130 (2008)

14. A Delabie, et al, App Phys Lett 91082904 (2007)

15. S Wang, K Kita, T Nishimura, K Nagashio, A Toriumi, Jpn J App Phys 50 04DA01 (2011)

16. S R M DiSilva, G K Rolim, G V Soares, I J R Baumvol, C Krug, L Miotti, F L Freire, C Radkte, App Phys Lett 100191907 (2012)

17. R Zhang, T Iwasaki, N Taoka, M Takenaka, S Takagi, App Phys Lett 98112902 (2011)

18. R Zhang, P C Huang, J C Lin, M Takenaka, S Takagi, App Phys Lett 102081903 (2013); IEEE Trans ED 60927 (2013)

19. S Swaminathan, M Shandalov, Y Oshima, P McIntyre, Appl. Phys Lett 96082904 (2010)

20. H Li, L Lin, J Robertson, App Phys Lett 101052903 (2012)

21. J Robertson, Y Guo, L Lin, J App Phys 117112806 (2015)

22. M L Huang, Y C Chang, C H Chang, Y J Lee, P Chang, J Kwo, T B Wu, M Hong, App Phys Lett 87252104 (2005)

23. Y Yuan, P D Ye, Electron Device Lett 29294 (2008)

24. R Suzuki, N Taoka, M Yokoyama, S Lee, S H Kim, T Hshi, T Yasuda, W Jevasuwan, T Maeda, O Ichikawa, N Fukuhara, M Hata, M Takenaka, S Takagi, App Phys Lett 100 132906 (2012)

25. L K Chu, C Merkling, A Alian, J Dekoster, J Kwo, M Hong, M Caymax, M Heyns, App Phys Lett 99042908 (2011)

26. T Hoshii, S Lee, R Suzuki, N Taoka, M Yokohama, H Yamada, M Hata, T Yasuda, M Takenaka, S Takagi, J App Phys 112073702 (2012)

27. D Colleoni, A Pasquarello, App Phys Lett 103142108 (2013)

28. A Stesmans, S Nguyen, V V Afanasev, App Phys Lett 103162111 (2013)

29. M Heyns, et al, Tech Digest IEDM (2011)

30. J Franco, B Kaczer, J Roussel, J Mitard, M Cho, L Witters, T Grasser, G Groeseneken, IEEE Trans ED 60396 (2013)

31. J Franco, et al, Proc IRPS (IEEE, 2014)

32. G Groeseneken et al, Tech Digest IEDM (2014) p34.4 
33. E J Kim, L Q Wang, P M Ashbeck, K C Saraswat, P C McIntyre, App Phys Lett 96012906 (2010)

34. Y Yuan, B Yu, J Ahn, P C McIntyre, P M Asbeck, M Rodwell, J Taur, IEEE Trans ED 59 $2100(2012)$

35. S. J. Clark and J. Robertson, Phys. Rev. B 82, 085208 (2010).

36. S. Lany and A. Zunger, Phys. Rev. B 78, 235104 (2008).

37. J Robertson, Adv Phys 32361 (1983)

38. P Lamparter, R Kniep, Physica B 234405 (1997)

39. C Landron, L Hennet, T E Jenkins, G N Greaves, J P Coutures, A K Soper, Phys Rev Lett 864839 (2001)

40. V V Afanasev, A Stesmans, J App Phys 102081301 (2007)

41. G Gutierrez, B Johnansson, Phys Rev B 65104202 (2002)

42. C G van de Walle, M Choi, J R Weber, J L Lyons, A Janotti, Microelec Eng 109211 (2013);

43. M Choi, A Janotti, C G van de Walle, J App Phys 113044501 (2013)

44. B Olliver, R Betoux, P Lacorre, D Massiot, G Ferey, J Mater Chem 71049 (1997)

45. Y Yourdshahyan, C Ruberto, L Bengtsson, B I Lundqvist, Phys Rev B 568443 (1997)

46. D Liu, S J Clark, J Robertson, App Phys Lett 96032905 (2010)

47. S Geller, J Chem Phys 33676 (1960)

48. C K Lee, E Cho, H S Lee, K S Seol, S Han, Phys Rev B 76245110 (2007)

49. K Chen, D A Drabold, J App Phys 919743 (2002)

50. J Robertson, J Vac Sci Technol A 31050821 (2013)

51. L Lin, J Robertson, App Phys Lett 98082903 (2011)

52. L Lin, J Robertson, J Vac Sci Technol B 30 04E101 (2012)

53. E P O’Reilly, J Robertson, Phys Rev B 34, 8684 (1986)

54. D Colleoni, A Pasquarello, J Phys Cond Mat 26492202 (2014)

55. Y Guo, L Lin, J Robertson, App Phys Lett 102091606 (2013) N

56. T Aoki, N Fukuhara, T Osada, H Azawa, M Hata, T Inoue, App Phys Lett 105033513 (2014)

57. Q H Luc, H B Do, T H Ha, C C Hu, Y C Lin, E Y Chang, IEEE ED Letts 361277 (2015)

58. $\quad$ R Gillen, J Robertson, J Phys Cond Mat 25405501 (2013)

59. J B Varley, A Janotti, C G van der Walle, Phys Rev B 93161201 (2016)

60. E Fois, A Selloni, G Pastore, Q M Zhang, R Car, Phys Rev B 4513378 (1992)

61. G V Soares, K P Bastos, R P Pezzi, L Miotti, C Driemeier, I J R Baumvol, C Hinkle, G Lucovsky, App Phys Lett 844992 (2004) 


\section{Figure Captions}

1. (a) Schematic of the PBTI reliability vs effective oxide thickness (EOT) in Si pMOS with $\mathrm{HfO}_{2}$ gate dielectric, and lower reliability of $\mathrm{Al}_{2} \mathrm{O}_{3}$ based gate stacks based on Ge and III-V channels. The reliability is expressed on the y axis as the maximum gate voltage that can be continuously applied that to cause a $30 \mathrm{mV} \mathrm{V}$ shift over a lifetime of 10 years. Adapted from refs [31,32]. (b) Schematic of the energy distribution of the defect levels causing the electron trapping in the gate oxide, deduced from the voltage dependence of the charge trapping kinetics of PBTI. Adapted from refs [31,32].

2. Defect formation energies of (a) oxygen vacancies in $\theta-\mathrm{Al}_{2} \mathrm{O}_{3}$, and (b) $\mathrm{N}$ vacancies in wurzite AlN, for anion rich chemical potential, calculated in the sX exchange correlation potential. The slopes of the lines are the defect charge state, which is indicated on the diagram. The stable charge density at any given Fermi energy is that with the lowest formation energy. The transition energy is where the stable charge changes.

3. Summary of defect transition energies between charge states, for $\mathrm{O}$ vacancies in $\theta-\mathrm{Al}_{2} \mathrm{O}_{3}, \mathrm{O}$ vacancies in $\kappa-\mathrm{Al}_{2} \mathrm{O}_{3}$ (from Choi [43]), and for the $\mathrm{N}$ vacancy in wurzite AlN. The defect charge states are indicated.

4. (a) A 96 atom random network model of amorphous $\mathrm{AlN}$ with no defects $(\mathrm{Al}=$ pink balls, nitrogen = blue). (b) Partial density of states in the a-AlN model, on both 4-fold and 3-fold coordinated sites.

5. (a) Partial density of states on the empty defect level (defect 1) and filled defect level (defect 2) referenced to the bulk PDOS. (b) a-AIN network with one $\mathrm{N}$ vacancy introduced, plus one hydrogen atom for charge counting.

6. (a) A random network model of $\mathrm{AlON}_{\mathrm{x}}$ with vacancy. (b) Calculated partial density of states on $\mathrm{Al}$ sites adjacent to $\mathrm{O}$ vacancies in $\mathrm{AlON}_{\mathrm{x}}$, calculated in $\mathrm{sX}$, and aligned against the Ge band edges using known band offsets. Note the defect DOS peaks lie away from the Ge band gap. (c,d) Calculated vacancy wavefunctions for the oxy-nitride network, (c) filled state in valence band, $(\mathrm{d})$ empty states in conduction band $(\mathrm{Al}=$ pink balls, oxygen $=$ red, nitrogen $=$ blue). 


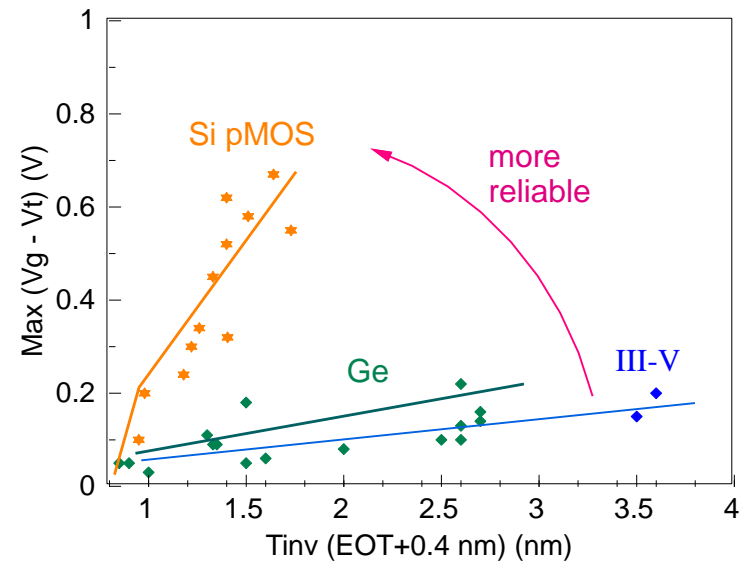

(a)

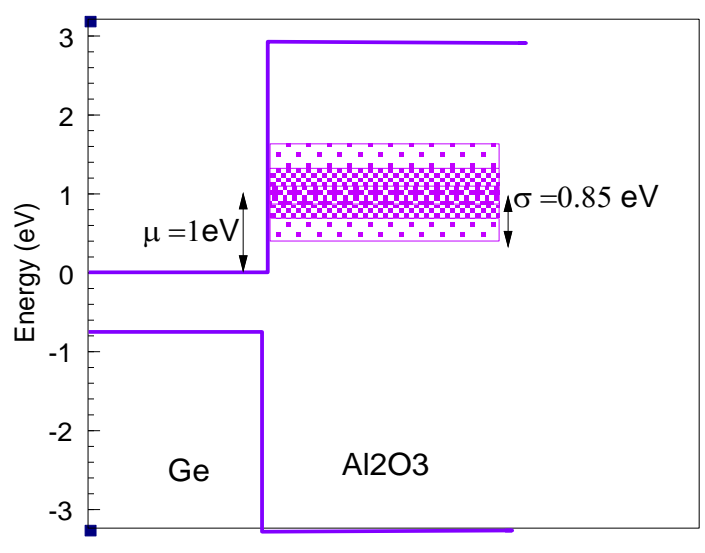

(b)

Fig. 1. 


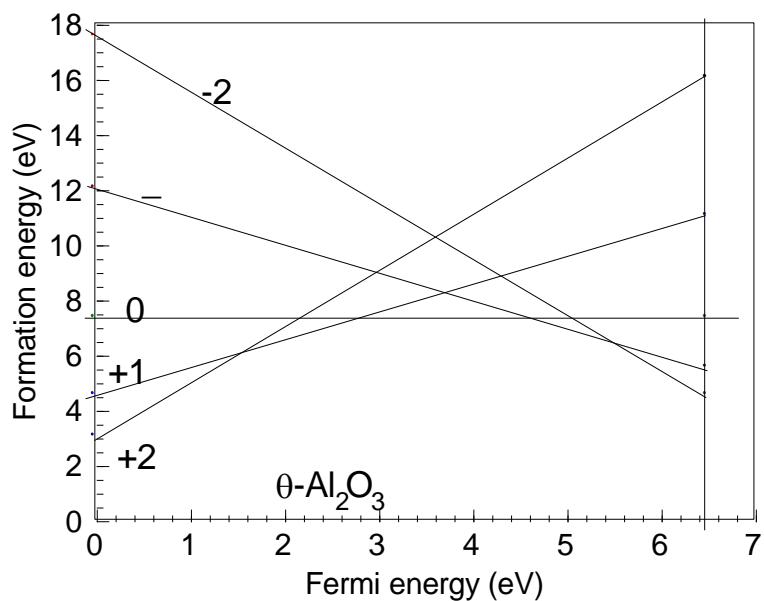

(a)

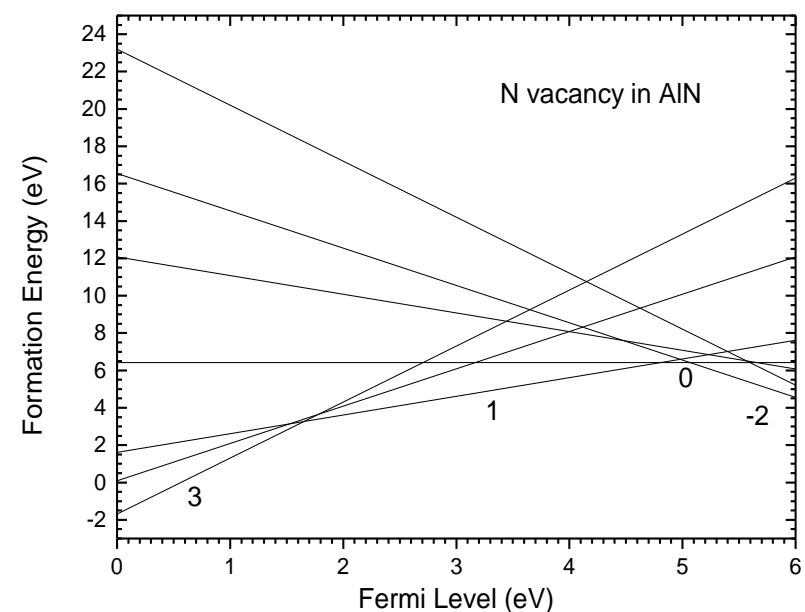

(b)

Fig. 2. 


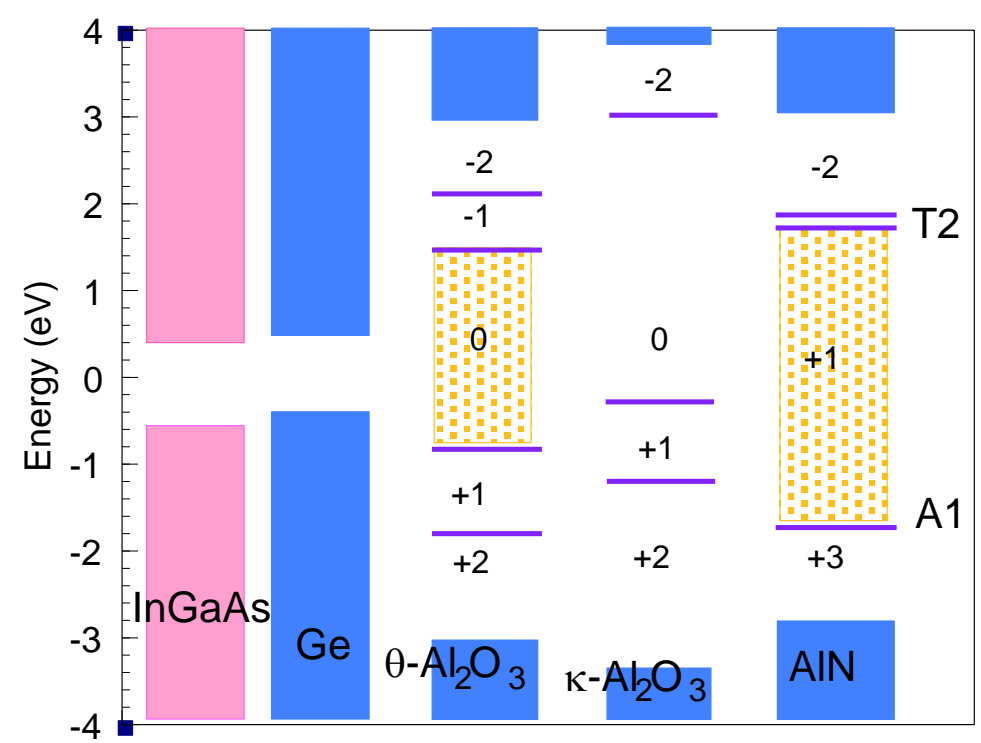

Fig. 3. 
(a)
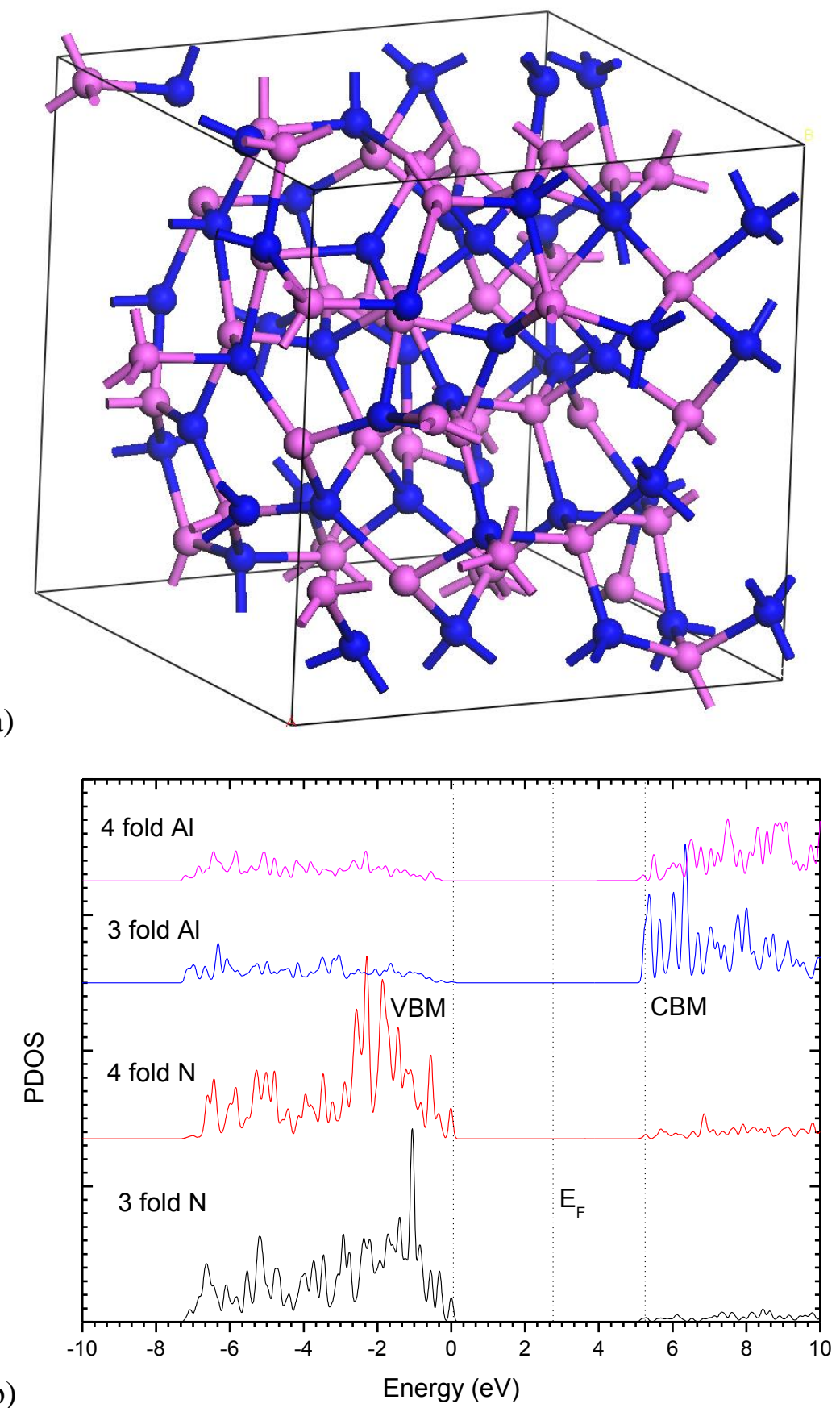

Fig. 4 


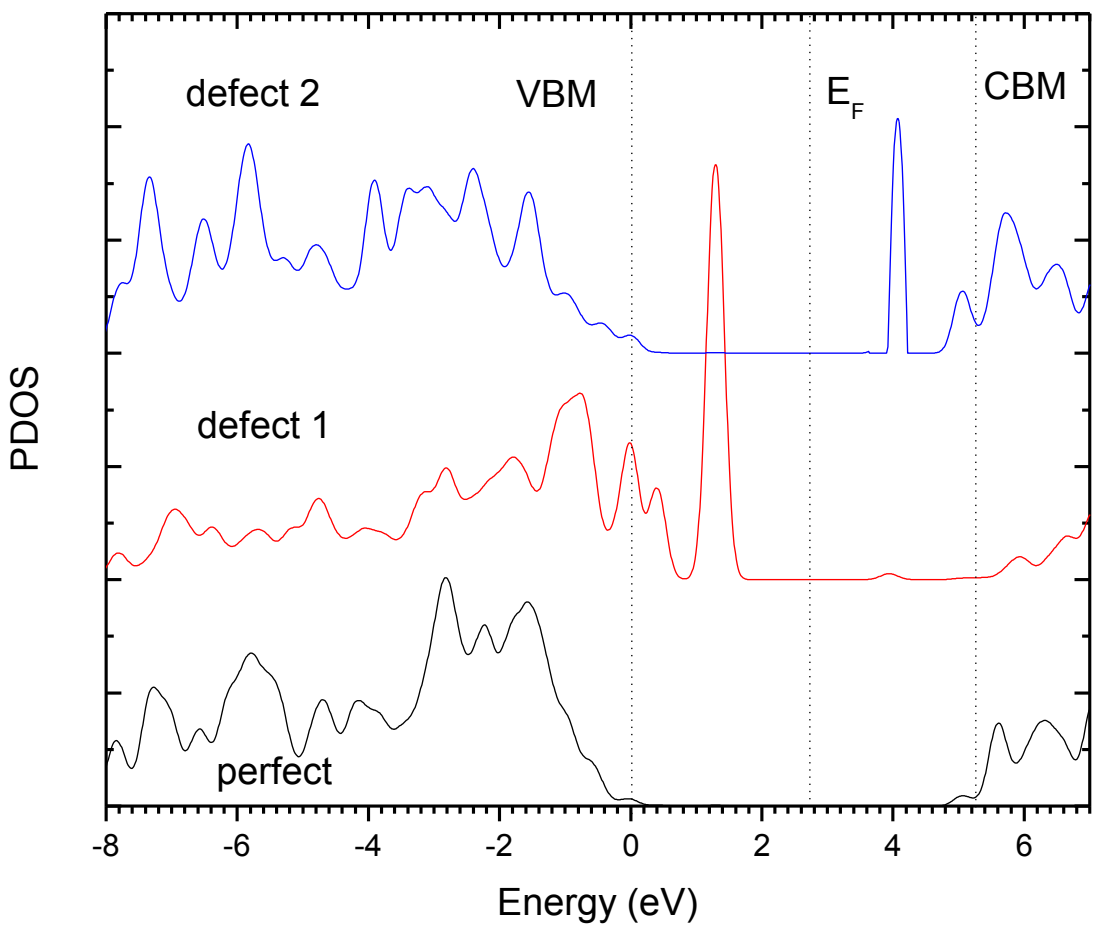

(a)

(b)

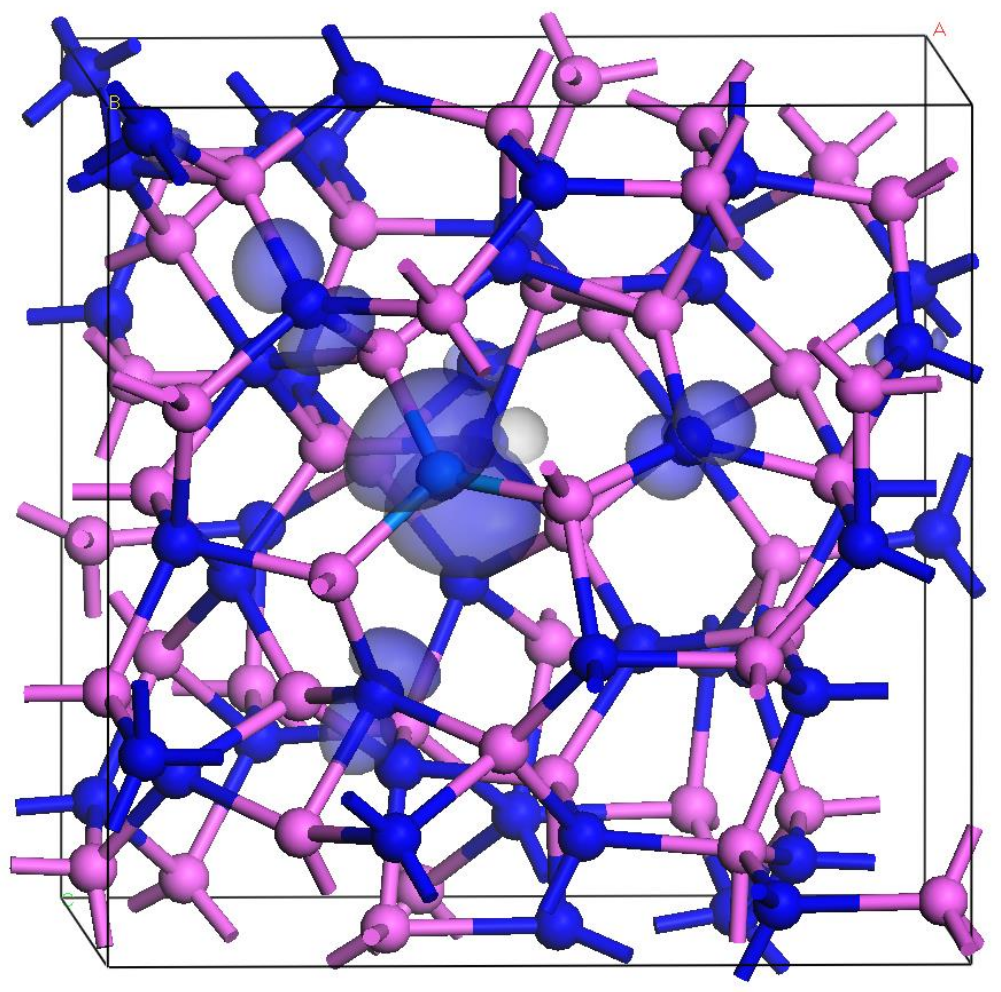

Fig. 5. 


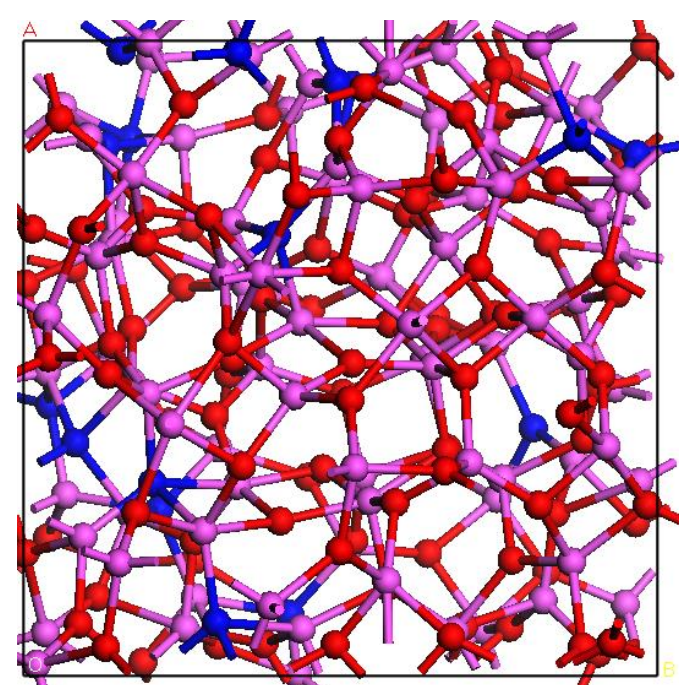

(a)

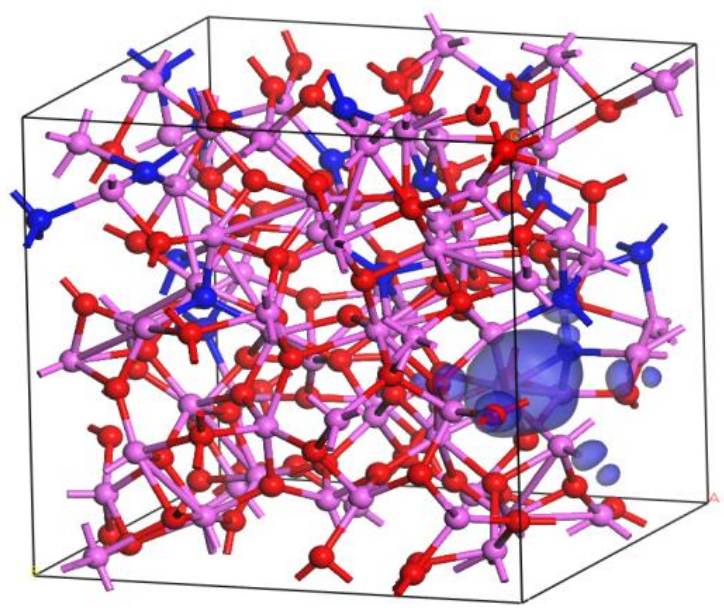

(c)

Fig. 6.

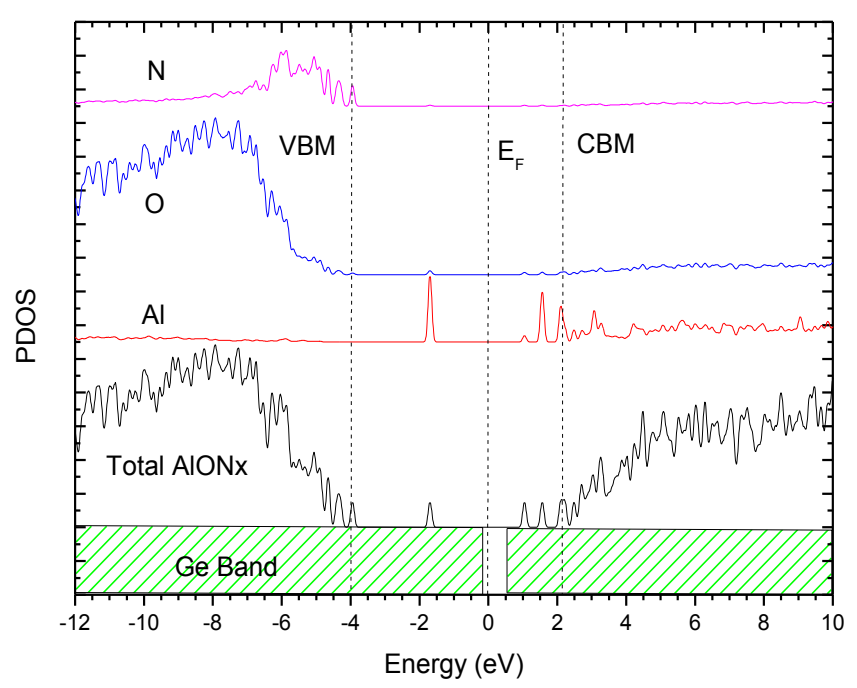

(b)

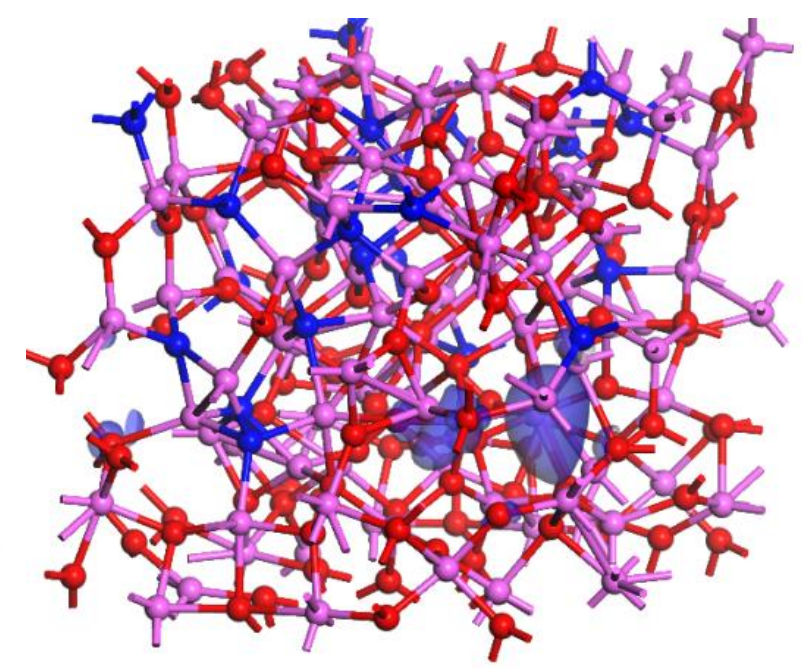

(d) 